BULLETIN OF THE

AMERICAN MATHEMATICAL SOCIETY

Volume 77, Number 2, March 1971

\title{
GENERATION OF EQUICONTINUOUS SEMIGROUPS BY HERMITIAN AND SECTORIAL OPERATORS. I
}

\author{
BY ROBERT T. MOORE ${ }^{1}$
}

Communicated by M. H. Protter, September 25, 1970

1. Introduction. This announcement and its sequel [8] conclude a series, beginning with [6] and [7], in which classical Banach-algebraic techniques are adapted to treat problems in operator theory on a general locally convex space (lcs). These announcements provide a representative sampling of the results which will appear, with full proofs and examples, in [9].

The material discussed below is concerned with the more geometrical aspects of the generation and perturbation theory of continuous (and/or holomorphic) semigroups of operators on an lcs. It unifies and extends several earlier lines of development: the HilleYosida generation theory and Phillips perturbation theory for $C_{0}$ semigroups on $B$-spaces [1], the norm-estimate theory of holomorphic semigroups on $B$-spaces developed by Hille and Phillips [1], and the geometric theory of hermitian (selfadjoint) and sectorial generators of unitary groups and holomorphic semigroups respectively (due respectively to Stone [10] and Kato [2] for Hilbert spaces, and to Lumer-Phillips [3] and the author [5] for $B$-spaces). Applications are illustrated in $\$ 5$ in the nonclassical setting of various differential operators and evolution equations on test function spaces.

The setting of the present announcement is that of a "calibrated" lcs $X$ with a "Lumer geometry" as described in [6] and [7]. By contrast, [8] is concerned with the more topological aspects of the theory, relating earlier work of Schwartz, Yosida, and Komatsu (see [11]) and the theory of "distribution semigroups" to the results discussed below. A primitive version of some of this material was sketched in [5], and references 6 and 7 cited there have been absorbed into the monograph [9].

AMS 1969 subject classifications. Primary 4750, 4748; Secondary 4601.

Key words and phrases. $C_{0}$ equicontinuous (semi)group, equicontinuous holomorphic semigroup, infinitesimal generator, semi-inner product, numerical range of an operator, sectorial operator, hermitian operator, Phillips adjoint of an operator, adjoint semigroup, perturbation of semigroup-generators, locally convex topological vector space.

1 The author gratefully acknowledges partial support of this research by the National Science Foundation, through grants GP 5585, 8964, and 12548. 
2. Sectorial operators and holomorphic semigroups. Here $\mathfrak{X}$ will denote a complex lcs, assumed throughout to be complete (some results are true in greater generality). A calibration $\Gamma$ for $\mathfrak{X}$ is a collection of seminorms $p$ which together induce the topology of $\mathfrak{X}$. For each angle $0 \leqq \Phi \leqq \pi / 2$, we regard the symmetric closed sector

$$
S_{\Phi}=\{z \in C:|\arg z| \leqq \Phi\}
$$

as an additive topological semigroup with the usual topology.

Definition 1. (a) A family $\left\{T_{z}: z \in S_{\Phi}\right\} \subset \mathscr{L}(\mathfrak{X})$ of continuous linear operators is a holomorphic semigroup of class $\Phi$ or is in $H(\Phi)$, iff

(i) the map $z \rightarrow T_{z}$ is a semigroup homomorphism into the multiplicative semigroup of $\mathscr{L}(\mathfrak{X})$ that carries 0 in to $I$ and is weak-operator continuous, and

(ii) on the interior $\operatorname{int}\left(S_{\Phi}\right)$ the map is weak-operator holomorphic. (That is, for all $u \in \mathfrak{X}$ and $u_{*}$ in the dual $\mathfrak{X}^{*}$, the map $z \rightarrow\left\langle T_{z} u, u_{*}\right\rangle \in \boldsymbol{C}$ is respectively continuous or holomorphic on the sets specified.)

(b) If for some calibration $\Gamma$, every $T_{z}\left(z \in S_{\Phi}\right)$ is a contraction $\left(p\left(T_{z} u\right) \leqq p(u)\right.$ for all $\left.p \in \Gamma\right)$, the semigroup is in $C H(\Phi, \Gamma)$.

REMARK. Essentially the same argument as that used in Chapter 7 of [5] shows that every $C H(\Phi, \Gamma)$ semigroup is "simply continuous" or "strongly continuous" $\left(z \rightarrow T_{z} u\right.$ is continuous from $S_{\Phi}$ into $\mathfrak{X}$ for every $u \in \mathfrak{X})$. In the degenerate case $\Phi=0$, a $C H(0, \Gamma)$ semigroup turns out to be exactly a $C_{0}$ equicontinuous semigroup in the sense of Yosida [11]. (One uses Theorem 4 of [6].)

Definition 2. (a) Suppose that $\left\{T_{z}: z \in S_{\Phi}\right\}$ is in $H(\Phi)$. Then $u \in \mathfrak{X}$ is in the domain $D(A)$ of the (infinitesimal) generator $A$ of the semigroup iff there exists a vector $A u \in \mathfrak{X}$ such that

$$
\lim \left\{t^{-1}\left(T_{t} u-u\right): t \rightarrow 0 \text { in }[0, \infty)\right\}=A u ;
$$

then we say that $A$ generates the semigroup.

(b) The semigroup is smooth iff $D(A)=\mathfrak{X}$.

If $\mathfrak{X}$ is barreled, then the generator $A$ of a smooth semigroup is continuous. In the $B$-space setting, such "bounded" generators are very special, but smooth semigroups are typical of problems formulated on the proper locally convex space.

Generators of $C H(\Phi, \Gamma)$ semigroups can be characterized in terms of their numerical ranges as defined in [7]; we recall the main ideas here. If $\Gamma$ calibrates $\mathfrak{X}$, then the $\mathfrak{X}$-part $\Lambda_{0}$ of a Lumer geometry for $(\mathfrak{X}, \Gamma)$ is defined by selecting a suitable semi-inner product $[,]_{p}$ for each $p \in \Gamma$ and setting $\Lambda_{0}=\left\{[,]_{p}: p \in \Gamma\right\}$. Then the $\mathfrak{X}$-part of the numerical range of $A$ is defined to be

$$
W\left(A, \Lambda_{0}\right)=\left\{[A u, u]_{p}:(u, p) \in \mathfrak{X} \times \Gamma \text { and } p(u)=1\right\} .
$$


Using the natural dual calibration $\Gamma_{*}$ for $\mathfrak{X}^{*}$, one similarly defines an $\mathfrak{X}^{*}$-part $\Lambda_{1}=\left\{[,]_{q}: q \in \Gamma_{*}\right\}$ of a Lumer geometry and an $\mathfrak{X}^{*}$-part $W\left(A^{\odot}, \Lambda_{1}\right)$ of the numerical range, where $A^{\odot}$ denotes the Phillips adjoint of $A$. Then the Lumer geometry is $\Lambda_{*}=\Lambda_{0} \cup \Lambda_{1}$, and $\Lambda_{*}$-numerical range of $A$ is $W\left(A, \Lambda_{*}\right)=W\left(A, \Lambda_{0}\right) \cup W\left(A^{\odot}, \Lambda_{1}\right)$.

Definition 3. If $0 \leqq \Phi \leqq \pi / 2$, let

$$
\Delta_{\Phi}=\{z \in C: \pi / 2+\Phi \leqq \arg z \leqq 3 \pi / 2-\Phi\}
$$

be the sector "orthogonal to" $S_{\Phi}$ (in the sense that their boundaries are perpendicular). Then $A$ is $\Phi$-sectorial with respect to a geometry $\Lambda_{*}$ for $(\mathfrak{X}, \Gamma)$ iff $W\left(A, \Lambda_{*}\right) \subset \Delta_{\Phi}$.

THEOREM 1. Let $0 \leqq \Phi \leqq \pi / 2$. Then the following conditions on an operator $A$ on a calibrated lcs $(\mathfrak{X}, \Gamma)$ are equivalent.

(a) The domain $D(A)$ is dense and $A$ is $\Phi$-sectorial with respect to some geometry $\Lambda_{*}$ for $(\mathfrak{X}, \Gamma)$.

(b) The closure $\bar{A}$ of $A$ is a densely defined (dd) operator which is $\Phi$ sectorial with respect to every $\Lambda_{*}$ for $(\mathfrak{X}, \Gamma)$.

(c) The closure $\bar{A}$ is a dd operator with $\Gamma$-spectrum $\sigma_{\Gamma}(\bar{A}) \subset \Delta_{\Phi}$ and if $d_{\lambda}=\operatorname{dist}\left(\lambda, \Delta_{\Phi}\right)>0$ then $d_{\lambda}\left\|(\lambda-\bar{A})^{-1}\right\|_{\Gamma} \leqq 1$.

(d) The closure $\bar{A}$ generates a $C H(\Phi, \bar{\Gamma})$ semigroup $\left\{T_{z}: z \in S_{\Phi}\right\}$.

(e) (Equivalent only when $\Phi>0$.) There exists a semigroup homomorphism $T$ of $\operatorname{int}\left(S_{\Phi}\right)$ into the unit ball of the Banach algebra $\mathcal{F}_{\Gamma}(\mathfrak{X})$ such that

(i) $D(A) \supset \cup\left\{T_{z} \mathfrak{X}: z \in \operatorname{int}\left(S_{\Phi}\right)\right\}$ and the latter is dense in $\mathfrak{X}$, and

(ii) the function $z \rightarrow T_{z}$ is complex-differentiable on int $\left(S_{\Phi}\right)$ in the

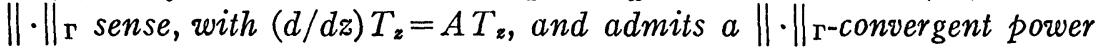
series expansion about each $z \in S_{\Phi}$.

Convention. In view of the equivalence of (a) and (b) above, we will simply speak of $A$ as " $\Phi$-sectorial with respect to $\Gamma$," regarding the arbitrary choice of some geometry $\Lambda_{*}$ as implicit.

The methods of proof are quite classical. For example, (a) and (b) are proved equivalent by means of Theorem 3 in [7] coupled with the method of Lemmas 3.3 and 3.4 in Lumer-Phillips [3]. Modulo [7], the remaining proofs can be built up from the $B$-space prototypes simply by using $\|\cdot\|_{\mathrm{r}}$ for the "operator norm".

We recall that $\mathfrak{X}^{\circ}$ is the strong $\left(\beta\left(\mathfrak{X}^{*}, \mathfrak{X}\right)\right)$ closure of the domain $D\left(A^{*}\right)$ of the adjoint of $A$ in $\mathfrak{X}^{*}[7]$. Let $\Gamma_{\odot}$ be the calibration on $\mathfrak{X}^{\odot}$ obtained by restriction from $\Gamma_{*}$.

THEOREM 2. Suppose A satisfies any one of the equivalent conditions in Theorem 1. Then the Phillips adjoint $A^{\odot}$ generates a $C H\left(\Phi, \Gamma_{\odot}\right)$ 
semigroup on $\mathfrak{X}^{\odot}$ whose values are just the restrictions of the adjoint semigroups $\left\{T_{z}^{*}: z \in S_{\Phi}\right\}$ to $\mathfrak{X}^{\odot}$. Consequently for any geometry $\Lambda_{*}$ for $\left(\mathfrak{X}^{\odot}, \Gamma_{\odot}\right), A^{\odot}$ is $\Phi$-sectorial.

In some applications, especially those involving nonsmooth semigroups where $D\left(A^{\odot}\right)$ is difficult to determine, direct calculation of the $\mathfrak{X}^{*}$-part $W\left(A^{\odot}, \Lambda_{1}\right)$ of the numerical range is highly impractical, and the following lemma is a more efficient test for $\Phi$-sectoriality.

Lemma 3. $A$ densely-defined operator $A$ is $\Phi$-sectorial with respect to $\Gamma$ if it satisfies the two conditions:

(a) the $\mathfrak{X}$-part $W\left(A, \Lambda_{0}\right) \subset \Delta_{\Phi}$ for some Lumer geometry $\Lambda_{*}$ for $(\mathfrak{X}, \Gamma)$, and

(b) the range $\left(\lambda_{0}-A\right) D(A)$ is dense in $\mathfrak{X}$ for some $\lambda_{0} \notin \Delta_{\Phi}$.

3. Hermitian operators and Stone's theorem.

Definition 4. A family $\left\{T_{t}: t \in R\right\} \subset \mathfrak{L}(\mathfrak{X})$ is a (weakly) $C_{0}$ group iff the families $\left\{T_{t}^{+}=T_{t}: t \in[0, \infty)\right\}$ and $\left\{T_{t}^{-}=T_{-t}: t \in[0, \infty)\right\}$ are $H(0)$ semigroups such that $T_{t}^{+} T_{t}^{-}=T_{t} T_{-t}=I$ for all $t \in[0, \infty)$. If for some calibration $\Gamma$ for $\mathfrak{X}$ every $T_{t}$ is a $\Gamma$-symmetry [6], the group is a generalized unitary group or is in $R C_{0}(\Gamma)$.

Definition 5. (a) The infinitesimal generator $A$ of a $C_{0}$ group is that of the positive semigroup $\left\{T_{t}^{+}=T_{t}: t \in[0, \infty)\right\}$, and the group is smooth if $D(A)=\mathfrak{X}$.

(b) An operator $H$ is hermitian with respect to a geometry $\Lambda_{*}$ for $(\mathfrak{X}, \Gamma)$ iff $W\left(H, \Lambda_{*}\right) \subset \boldsymbol{R}$.

THEOREM 4. Let $H$ be an operator on a calibrated lcs $(\mathfrak{X}, \Gamma)$. Then the following are equivalent.

(a) The domain $D(H)$ is dense in $\mathfrak{X}$ and $H$ is hermitian with respect to some geometry $\Lambda_{*}$ for $(\mathfrak{X}, \Gamma)$.

(b) The closure $\bar{H}$ of $H$ is a dd hermitian operator with respect to every $\Lambda_{*}$ for $(\mathfrak{X}, \Gamma)$.

(c) The closure $\bar{H}$ is a dd operator with $\sigma_{\Gamma}(H) \subset R$, and if $\operatorname{Im}(\lambda) \neq 0$, $|\operatorname{Im}(\lambda)|\left\|(\lambda-H)^{-1}\right\|_{\Gamma} \leqq 1$.

(d) The closure $A=(i H)^{-}=i H$ is the generator of a generalized unitary group.

The theorem follows easily from Theorem 1. (Notice that $H$ is hermitian iff $\pm i H$ are 0 -sectorial.) In the same way, one obtains the obvious analog of Theorem 2 for dual groups. An analog of Lemma 3 is also available.

Generalized unitary groups sometimes arise as boundary values of $C H(\pi / 2, \Gamma)$ semigroups. (If $A$ is $\pi / 2$-sectorial, $H=-i A$ is hermitian.)

4. Perturbation theorems. In the common setting of a reflexive 
calibrated lcs $(\mathfrak{X}, \Gamma)$, let us equip $\mathscr{L}(\mathfrak{X})$ with the topology of simple convergence to form $\mathscr{L}_{s}(\mathfrak{X})$, and pick out the topological subspace

$$
\mathcal{O}_{\Phi}(\Gamma)=\left\{A \in \mathfrak{L}_{s}(\mathfrak{X}): A \text { is } \Phi \text {-sectorial with respect to } \Gamma\right\}
$$

defined by $0 \leqq \Phi \leqq \pi / 2$. By our remarks after Definition 1 , the set $C H(\Phi, \Gamma)$ of holomorphic contraction semigroups can be viewed as a subset of $C\left(S_{\Phi}, \mathfrak{L}_{s}(\mathfrak{X})\right)$, the continuous functions from $S_{\Phi}$ to $\mathfrak{L}_{s}(\mathfrak{X})$, and can be endowed with the compact convergence topology.

TheOREM 5. (a) The set $\mathcal{O}_{\Phi}(\Gamma)$ is a closed cone in $\mathfrak{L}_{s}(\mathfrak{X})$.

(b) The map $A \rightarrow\left\{\right.$ "exp $\left.z A ": z \in S_{\Phi}\right\}=\left\{T_{z}: z \in S_{\Phi}\right\}$ from generators to semigroups is continuous from $\mathcal{\Theta}_{\Phi}(\Gamma)$ to $C H(\Phi, \Gamma) \subset C\left(S_{\Phi}, \mathfrak{L}_{s}(\mathfrak{X})\right)$.

A comparable theorem is true for generalized unitary groups: the map from the real closed lcs of hermitians to groups-as-functions-on $R$ is continuous.

5. Examples. Let $\mathfrak{X}$ be the space of $C^{\infty}$ functions on $R^{n}$ with period 1 along each coordinate axis (alias $C^{\infty}$ functions on the $n$-torus). For each $1 \leqq r \leqq \infty$, we calibrate $\mathfrak{X}$ with a "topology of $L^{r}$-convergence of derivatives", where $\|u\|_{r}=\left(\int_{K}|u|^{r} d x\right)^{1 / r}$ for $1 \leqq r<\infty$ and $\|u\|_{\infty}$ $=\sup \{|u(x)|: x \in K\}$ with $K$ the unit cube. Letting $D_{j}=\partial / \partial x_{j}$, define

$$
p_{r, m}(u)=\sum\left\{\left\|D_{1}^{\alpha(1)} \cdots D_{n}^{\alpha(n)} \cdots u\right\|_{r}^{2}: \alpha(1)+\cdots+\alpha(n) \leqq m\right\}^{1 / 2}
$$

and

$$
\Gamma(r)=\left\{p_{r, m}: m=0,1 \cdots\right\} .
$$

By a generalized Sobolev lemma, the $\Gamma(r)$ all calibrate the same nuclear Fréchet "test function" topology on $\mathfrak{X}$, with $r=1,2$ and $\infty$ of primary interest.

EXAMPLE 1. For every $1 \leqq r \leqq \infty$, the continuous operators $i D_{j}$ $\in \mathcal{L}(\mathfrak{X})$ can be shown by direct calculation to be $\Gamma(r)$-hermitian. As in Theorem 4 and the remark following Theorem 5 , every $D=\sum \alpha_{j} D_{j}$ generates a smooth generalized unitary group for $\Gamma(r)$ which translates functions in the direction $\left(\alpha_{i}, \cdots, \alpha_{n}\right)$, and this group varies continuously with respect to the real parameters $\alpha_{i}$.

Example 2. Various methods (e.g. $\$ 6$ of [8]) can be used to check that each $D_{j}^{2}$ is at least 0 -sectorial (dissipative) for every $\Gamma(r)$, $1 \leqq r \leqq \infty$. For $r=1$ and $r=\infty$, the numerical ranges can be shown to exhaust the entire left half-plane, while by contrast every $D_{j}^{2}$ is $\pi / 2$ sectorial with respect to $\Gamma(2)$, exhibiting the extreme sensitivity of the numerical ranges to choice of calibration. Thus for $\Gamma(2)$ (which has a 
unique $\mathfrak{X}$-part of a Lumer geometry consisting of inner products) every nonnegative combination $L=\sum \alpha_{j} D_{j}^{2}$ generates a smooth holomorphic contraction semigroup in the right half-plane, with generalized unitary boundary group. The choice $\alpha_{j}=1,1 \leqq j \leqq n$, yields the solution to the heat equation on the $n$-torus, without recourse to ellipticity or "deficiency" arguments. Results for $r \neq 1$, $2, \infty$ can be obtained by interpolation.

\section{REFERENCES}

1. E. Hille and R. S. Phillips, Functional analysis and semi-groups, rev. ed., Amer. Math. Soc. Colloq. Publ., vol. 31, Amer. Math. Soc., Providence, R. I., 1957. MR 19, 664.

2. T. Kato, Perturbation theory for linear operators, Die Grundlehren der math. Wissenschaften, Band 132, Springer-Verlag, New York, 1966, §V 3.10 and IX 1.6. MR 34 \#3324.

3. G. Lumer and R. S. Phillips, Dissipative operators in a Banach space, Pacific J. Math. 11 (1961), 679-698. MR 24 \#A2248.

4. Robert T. Moore, Measurable, continuous and smooth vectors for semigroups and group representations, Mem. Amer. Math. Soc. No. 78 (1968). MR 37 \#4669.

5. - Duality methods and perturbation of semigroups, Bull. Amer. Math. Soc. 73 (1967), 548-553. MR 36 \#5759.

6. - Banach algebras of operators on locally convex spaces, Bull. Amer. Math. Soc. 75 (1969), 68-73. MR 38 \#5018.

7. - Adjoints, numerical ranges, and spectra of operators on locally convex spaces, Bull. Amer. Math. Soc. 75 (1969), 85-90. MR 39 \#805.

8. - Generation of equicontinuous semigroups by hermitian and sectorial operators. II, Bull. Amer. Math. Soc. (to appear).

9. - Operator theory on locally convex spaces. I: Banach algebras, states, and numerical ranges (in prep.).

10. M. Stone, On one-parameter unitary groups in Hilbert space, Ann. of Math. 33 (1932), 643-648.

11. K. Yosida, Functional analysis, Die Grundlehren der math. Wissenschaften, Band 123, Academic Press, New York; Springer-Verlag, Berlin, 1965. MR 31 \#5054.

University of Washington, Seatrle, Washington 98105 